\title{
Implications of ELT observations of pulsars, anomalous X-ray pulsars and supernova remnants
}

\author{
Andrew Shearer, Pádraig O'Connor \\ and Seathrún Ó. Tuairisg \\ Computational Astrophysics Laboratory, Dept. of Information Technology, National University \\ of Ireland, Galway, Ireland
}

\begin{abstract}
Supernova remnants are the remains of the outer envelope of the supernova progenitor star. Neutron stars are one of the possible remnants of the region where the explosion detonated. Our knowledge, from galactic studies, of SNRs is well established as their morphology can be understood in terms of the initial blast and out-gassing during the last stages of the progenitor. However, observationally the link between neutron stars and SNRs is only poorly established. During a supernova there are a number of possibilities to produce a condensed remnant - no remnant; a neutron star (pulsar?); a magnetar; a black hole or something more exotic. We do not know what fraction of supernovae go down these possible paths. In the ELT era we will have the first real opportunity to sample the pulsar population in external galaxies and get a more comprehensive survey of optical emission from local pulsars. Such a survey would have significantly reduced biases compared to the current state of radio surveys particularly in the area of pulsar-SNR statistics. Furthermore, a $50 \mathrm{~m}$ telescope will be able to survey galaxies out to at least $20 \mathrm{Mpc}$ for young SNRs using $\mathrm{H} \alpha:[\mathrm{OIII}]$ and $\mathrm{H} \alpha$ :[SII] ratios. Currently there are over 1500 radio pulsars detected, 14 of which have been observed at optical wavelengths. Although small in number the family of optical pulsars yield much useful information in bridging the gap between the long wavelength radio emission and the high energy gamma-ray emission from pulsars. Specifically optical techniques are currently the only way of detecting polarisation in the high-energy regime. The advent of ELTs will increase the detection rate of local galactic pulsars and provides the possibility of detecting a significant number of extragalactic optical pulsars. Phenomenologically, the Pacini scaling law predicts $\sim 150$ galactic pulsars to have pulsed optical emission with an $\mathrm{m}_{V}<32$. Using SKA, Crab-like giant radio pulses should be detectable out to $7 \mathrm{Mpc}$. In contrast Crab-like pulsars would have a normal peak pulsed $\mathrm{m}_{V} \sim 31$ at $10 \mathrm{Mpc}$ making ELT optical observations more sensitive than radio observations and the best method for extragalactic pulsar discovery. To date only five AXPs have observed IR emission and two optical emission. ELTs will be able to sample the AXP population within the Galaxy as well as the local group again providing better statistics for the birth rate of AXPs compared to 'normal' pulsars. A combination of an AXP and 'normal' pulsar survey will make a significant contribution to the birthrate question - what fraction of supernovae produce pulsars compared to AXPs and other condensed objects?
\end{abstract}

Keywords. ISM: supernova remnants, stars: pulsars: general, stars: neutron

\section{Detecting optical pulsars using ELTs}

Pulsars are extremely stable emitters of periodic, pulsed radiation with periods in the range $1.56 \mathrm{~ms}-11.56 \mathrm{~s}$ (median $\sim 250 \mathrm{~ms}$ ) and period derivative ranges $10^{-12} \mathrm{ss}^{-1}<$ $\frac{d P}{d t}<10^{-22} \mathrm{ss}^{-1}$. There are currently over 1600 pulsars, all but six of which are radio observable and $\sim 95 \%$ of which are only radio observable. Extremely optically faint objects, the pulsar detection rate was only two a decade until 1994, and around one a year 
Table 1. Selected parameters of the 5 optical pulsars. The final two columns list their magnitudes were they at a distance of $1 \mathrm{Mpc}$ and the distances at which they would be with a visual magnitude of 30 (expected to be the limiting magnitude of a 50m ELT).

\begin{tabular}{lcccccc}
\hline Pulsar & Mag & Dist.(pc) & Period & Av & Mag @1Mpc & Dist. at 30mag \\
\hline Crab & 16 & 1700 & 33 & 1.6 & 33 & $813 \mathrm{kpc}$ \\
PSR 0540-69 & 23 & 230 & 50 & 0.2 & 32 & $1.9 \mathrm{Mpc}$ \\
PSR B0833+45 (Vela) & 24 & 49400 & 89 & 0.6 & 46 & $4.3 \mathrm{kpc}$ \\
PSR 0656+14 & 25 & 160 & 385 & 0.09 & 47 & $2900 \mathrm{pc}$ \\
Geminga & 26 & 290 & 237 & 0.07 & 49 & $1270 \mathrm{pc}$ \\
\hline
\end{tabular}

since, mainly due to the high sensitivity of the FOC and STIS. All these post-1994 objects have $\mathrm{m}_{V}<25$ and all but three have been detected by the HST. Because of their intrinsic faintness, most have been detected due to small ISM absorption and their relative nearness. There are currently 14 known pulsars with optical emission, of which five exhibit optical pulsations.

The phenomenological model of Pacini et al. (1971), based on synchrotron radiation from a power law spectrum of particles within the pulsar magnetosphere radiating at a constant fraction of the light cylinder, has been the most successful for describing the pulsed optical luminosity. It is a scaling model which parameterizes emission as a function of known pulsar parameters, with pulsed luminosity scaled as:

$$
\begin{aligned}
& F_{\nu, \text { thin }} \propto B^{4-\alpha} P^{2 \alpha-9} \psi^{2-\alpha} \delta^{-1}, \\
& F_{\nu, \text { thick }} \propto B^{13 / 3} P^{-10} \psi^{7 / 3} \delta^{1-n},
\end{aligned}
$$

where $B=$ surface magnetic field, $P=$ pulsar period, $\psi=$ particle pitch angle, $\delta=$ pulsar duty cycle and $\alpha=$ photon spectral index at optical wavelengths.

Pacini scaling predicts $\sim 35$ objects should have optical emission at $\mathrm{m}_{V}<27$ and $\sim 130$ objects should have optical emission at $\mathrm{m}_{V}<32$. This suggests that there remain many more to be detected optically, the faintest known optical pulsars testing the limits of the current telescopes.

The importance of building up a spectral energy distribution of pulsars cannot be underestimated. At infrared wavelengths the Crab pulsar shows indications of a 'rollover' in its non-thermal spectrum. As optical emission is generally assumed to be due to synchrotron radiation, this could possibly be due to (i) a low energy cut-off in the particle distribution (ii) particles emitting below their critical frequency or (iii) synchrotron selfabsorption. If due to self-absorption we can parameterize emission in a similar sense to the Pacini model (O'Connor, Golden \& Shearer 2005) and obtain a scaling relationship for the rollover frequency:

$$
\nu_{\text {rollover }} \propto P^{(-31-10 \alpha)(2 \alpha+5)} P^{(7+2 \alpha)(2 \alpha+5)}
$$

This rollover occurs at infrared wavelengths for most pulsars. Currently, the Crab pulsar is the only object for which sufficient data are available to suggest evidence for synchrotron self-absorption - larger telescopes will allow us to quantify SSA for other fainter pulsars.

The advent of ELTs will allow a much deeper probe of the local neighbourhood and provides the possibility of detecting a significant number of extragalactic optical pulsars. For instance, a telescope like the Euro50 could observe Crab-like objects further than a distance of $\sim 1 \mathrm{Mpc}$, and provide concrete magnitudes for our fainter neighbours. The 

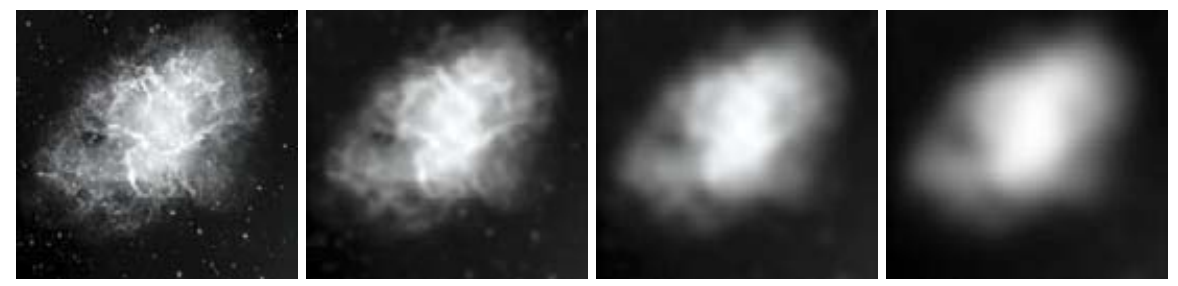

Figure 1. The change in spatial resolution as the Crab is moved further away. For each image, from left to right, the distances are doubled. The original image is from the VLT.

identification of extragalactic radio pulsars (for example by SKA) can facilitate follow up ELT observations to search for the optical counterparts to these objects.

\subsection{Optical signatures of Anomalous X-ray Pulsars}

AXPs, X-ray sources with long (6-12s) pulse periods, are 'anomalous' because the power associated with rotational energy loss through spin-down is not sufficient to produce the observed luminosities and no binary companions are seen. Soft Gamma-ray Repeaters (SGRs) are objects which give sporadic bursts of hard X-ray/soft $\gamma$-rays as well as rare, very luminous $\left(\sim 10^{44} \mathrm{erg}\right)$ giant flares. With similar periods and period derivatives to AXPs, they are thought to be young objects from their spin-down and from SNR associations (in some cases). The two groups have been linked by the discovery of persistent emission from SGRs that is similar to AXPs. SGR-like bursts, with fast rise times, short durations and hard spectra relative to the quiescent emission were detected from two AXPs. AXPs and SGRs suggest a very different type of neutron star to the radio pulsars and X-ray pulsars in binaries.

AXP models were heavily based on their X-ray emission, but most models became untenable with the discovery of optical and IR counterparts (Hulleman et al. 2000). In the currently accepted magnetar model (Thompson \& Duncan 1996) they are neutron stars with dipole magnetic fields of order $\mathrm{B} \sim 10^{14}-10^{15} \mathrm{G}$, the decay of which supplies the energy of the observed luminance. Their optical emission is most likely magnetospheric in origin. Hulleman et al. (2004) produced a spectral energy distribution of 4 U $0142+61$ showing a sharp break in the optical (unique among neutron star objects) and Kern \& Martin (2002) discovered optical pulsations, modulated at the X-ray period. Five AXPs have been observed at IR wavelengths and there are suggestions of IR variability from AXP 1E 1048.1-5937 (Durant \& van Kerkwijk 2005).

Only recently has it been possible to construct broad Spectral Energy Distributions (SEDs) for the AXPs. Increased detections in the optical and infrared make it possible to examine flux ratios $\left(F_{X} / F_{I R}, F_{X} / F_{V}\right)$ and spectral features within the SEDs for the first time. Most AXPs are too highly reddened to be detected in $V$ or $B$ but ELTs can address these issues (in a similar fashion to 'normal' pulsars). Other AXPs could be analysed for optical spectral breaks (as in Hulleman et al. 2004) and for IR variability, providing constraints and refinements to models. The smaller population, the larger associated reddening and the limited SED extent of AXPs (c.f. the normal optical pulsars) means that ELTs can contribute significantly to our knowledge of AXPs.

\section{Observing supernova remnants with ELTs}

Supernova remnants (SNRs) and their interaction with their local environment dictate the dynamics of the ISM, play a significant role in the provenance of cosmic rays and provide a direct measure of the supernova rate, the massive star formation rate and the 
galactic high-end initial mass function. Recent SNR surveys targeted nearby galaxies with favourable inclination, including M33 (Gordon et al. 1998, Ghavamian et al. 2005), M31 (Magnier et al. 1995) and M83 (Blair \& Long 2004). Incompleteness in these surveys is rife. For example, Blair \& Long detected 71 SNRs in M83. Historically, 6 SNe were seen since 1923, giving a SN rate of $\sim 1$ every 15 years, implying it contains above 500 SNRs (assuming a 10000-year lifetime). Source confusion, in particular where high [SII]:H $\alpha$ regions (indicative of SNRs) superimposed on HII regions are difficult to detect. SNRs in OB associations create large superbubbles, where several SNe occur in the $10^{7}$-year lifetime of a typical massive star, making it difficult to distinguish individual older SNRs. Wind-shocked nebulae (with large $[\mathrm{SII}]: \mathrm{H} \alpha$ ratios) further contaminate surveys by up to $14 \%$ (Braun \& Walterbos 1993). With age, SNRs increase in size and decrease in brightness, making it difficult for even large telescopes to detect them. Expecting a larger number of fainter, large-diameter SNRs, Matonick \& Fesen (1997) estimated the incompleteness (which was considerable) in their SNR surveys by constructing histograms of their findings in brightness and size.

The apparent angular diameter the Crab SNR (2.4 pc across) at $10 \mathrm{Mpc}$ is $0^{\prime \prime} .05$. With a diffraction limit of a $50 \mathrm{~m}$ telescope of $0^{\prime \prime} .0025$, a 'typical' SNR is resolvable. However AO limitations in the optical restrict SNR observations for the foreseeable future to seeinglimited resolutions, at best around $0^{\prime \prime} .4$. This does not preclude good scientific returns on observations, however, as we would have the larger FoV necessary for serendipitous discoveries from large-scale surveys. Furthermore, the increase in light-gathering power of ELTs would allow the fainter (older, large-diameter) SNR population to be more comprehensively examined. The greater spatial resolution can resolve structure, easier distinguish SNRs within superbubbles and isolate HII regions and planetary nebulae.

We also have much to gain from detailed observations of plerions, where energy input is supplied from a pulsar wind. Other than the Crab and PSR B0540-69, we have data on plerion emission only at $\mathrm{cm}$ wavelengths and in X-rays below about $10 \mathrm{keV}$. ELTs would be ideally positioned to image individual plerions at great distances. A typical VLT image of the Crab (such as that shown above), with a seeing of $0^{\prime \prime} .6$ and an angular size of $6^{\prime} .8 \times$ $6^{\prime} .8$ is 628 resolution elements across. An ELT $(50 \mathrm{~m})$ image with the equivalent number of resolution elements is $1^{\prime \prime} .57$ in diameter (for diffraction-limited optics). This corresponds to a distance of $\sim 300 \mathrm{kpc}$ for an object the diameter of the Crab nebula, essentially achieving VLT-like spatial resolution at this distance. High resolution observations of features like wisps and knots (interpreted as polar and equatorial zones of the magnetized collisionless shock terminating the pulsar wind) help constrain theoretical models of pulsars. Detailed analyses of bow-shocks can provide information on the pulsar's velocity vector with respect to the line-of-sight, and hence trace it back to its progenitor stellar population.

\section{References}

Blair, W.P. \& Long, K.S. 2004, ApJS 155, 101

Braun, R. \& Walterbos, R.A.M. 1993, A\&AS 98, 327

Durant, M. \& van Kerkwijk, M.H. 2005, ApJ 627, 376

Ghavamian, P., Blair, W.P., Long, K.S., Sasaki, M., Gaetz, T.J. \& Plucinsky, P.P. 2005, AJ 130, 539

Gordon, S.M., Kirshner, R.P., Long, K.S., Blair, W.P., Duric, N. \& Smith R.C. 1998, ApJS 117,89

Hulleman, F., van Kerkwijk, M.H. \& Kulkarni, S.R. 2004, A\& A 416, 1037

Hulleman, F., van Kerkwijk, M.H. \& Kulkarni, S.R. 2000, Nature 408, 689

Kern, B. \& Martin, C. 2002, Nature 417, 527 
Magnier, E.A., Prins, S., van Paradijs, J., Lewin, W.H.G., Supper, R., Hasinger, G., Pietsch, W. \& Truemper, J. 1995, A\&AS 114, 215

Matonick, D.M., Fesen, R.A. 1997, ApJS 112, 49

O'Connor, P., Golden, A. \& Shearer A. 2005, ApJ 631, 471

Pacini, F. 1971, ApJ Letters 163, L17

Thompson, C. \& Duncan, R.C. 1996, ApJ 473, 322

\section{Discussion}

BuCKLEY: Can you comment on the proposition that AXPs are a manifestation of Thorne-Zytkow objects?

TUAIRISG: Although the estimated birthrate of AXPs is consistent with that of ThorneZytkow objects, the recent detection of optical counterparts makes the magnetar model a more likely one.

DRAVINS: You showed predictions of apparent optical brightness for the expected pulsar population in the Galaxy. Are there any similar predictions for, specifically, millisecond pulsars?

TUAIRISG: The numbers quoted were obtained by applying Pacini's scaling law to all radio pulsars in the Galaxy, regardless of period. Applying this only to millsecond pulsars would obtain those expected optical brightnesses.

BAADE: A particularly attractive subject in this domain should be the search for remnants of gamma-ray bursts.

TUAIRISG: Indeed, ELTs would be perfectly positioned to capture low-magnitude transient optical counterparts, and image for a longer duration than conventional telescopes. 